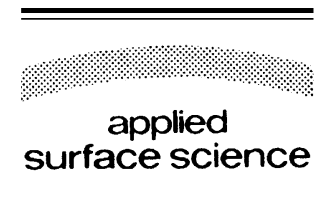

Applied Surface Science 228 (2004) 143-150

\title{
Field penetration induced charge redistribution effects on the field emission properties of carbon nanotubes - a first-principle study
}

\author{
Chun-Wei Chen ${ }^{\mathrm{a},{ }^{*}}$, Ming-Hsien Lee ${ }^{\mathrm{b}}$, S.J. Clark ${ }^{\mathrm{c}}$ \\ ${ }^{a}$ Department of Materials Science and Engineering, National Taiwan University, 1 Roosevelt Road, Sector 4, \\ Taipei 106, Taiwan, ROC \\ ${ }^{\mathrm{b}}$ Department of Physics, Tamkang University, Tamsui, Taipei, Taiwan, ROC \\ ${ }^{\mathrm{c}}$ Department of Physics, University of Durham, Durham DH1 3LE, UK
}

Received 17 October 2003; received in revised form 3 January 2004; accepted 3 January 2004

\begin{abstract}
The effect of field penetration induced charge redistribution on the field emission properties of carbon nanotubes (CNTs) have been studied by the first-principle calculations. It is found that the carbon nanotube becomes polarized under external electric field leading to a charge redistribution. The resulting band bending induced by field penetration into the nanotube tip surface can further reduce the effective workfunction of the carbon nanotubes. The magnitude of the redistributed charge $\Delta Q$ is found to be nearly linear to the applied external field strength. In addition, we found that the capped $(9,0)$ zigzag nanotube demonstrates better field emission properties than the capped $(5,5)$ armchair nanotube due to the fact that the charge redistribution of $\pi$ electrons along the zigzag-like tube axis is easier than for the armchair-like tube. The density of states (DOS) of the capped region of the nanotube is found to be enhanced with a value $30 \%$ higher than that of the sidewall part for the capped $(5,5)$ nanotube and $40 \%$ for the capped $(9,0)$ nanotube under an electric field of $0.33 \mathrm{~V} / \AA$. Such enhancements of the DOS at the carbon nanotube tip show that electrons near the Fermi level will emit more easily due to the change of the surface band structure resulting from the field penetration in a high field.
\end{abstract}

(C) 2004 Elsevier B.V. All rights reserved.

Keywords: Field emission; Carbon nanotubes; First-principle; Workfunction

\section{Introduction}

Carbon nanotubes (CNTs) have attracted considerable attention due to their unique geometry and interesting electronic properties, which suggest potential applications in field emission displays [1] and other vacuum microelectronic devices [2]. Their highaspect ratio leads to a large electric field enhancement

\footnotetext{
* Corresponding author. Tel.: +886-2-33665205; fax: $+886-2-23634562$.

E-mail address: chunwei@ntu.edu.tw (C.-W. Chen).
}

and a low emission threshold voltage. Up to now, most field emission studies on carbon nanotubes have described the emission process using Fowler-Nordheim $(\mathrm{F}-\mathrm{N})$ tunneling theory [3], suggesting that emission occurs by the quantum mechanical tunneling of electrons through high-aspect ratio carbon nanomaterials, with a potential barrier similar to that of graphite. It is well known from experiments as well as band structure calculations that the electronic structure of the nanotube depends strongly on its geometrical parameters, such as the diameter of the tube and its helicity [4]. Both metallic and semiconducting 
tubes exist. The tip of a carbon nanotube has different properties from those encountered along the nanotube sidewall [5]. Thus a single nanotube can be made with metallic and/or semiconducting parts. The typical Fowler-Nordheim tunneling theory, which usually describes the emitter as a perfect conductor, has been successful in describing the field emission from micron-sized tips. However, many experiments reflect that the F-N theory is not sufficient for a full understanding of the field emission mechanism for nanotubes. For example, nonlinear current-voltage characteristics occur in F-N plots [6]. Field emission energy distributions (FEED) of electrons emitted from individual multiwalled carbon nanotubes measured by Frasen et al. [7] indicate that the position of the peaks in the spectrum depends linearly on the extraction voltage, unlike metallic emitters, where the position stays in the vicinity of the Fermi level. These results are incompatible with the picture that the emission current originates purely from the usual metallic (extended) states, suggesting that localized states on the nanotube tip [8] may contribute to the emission current significantly. Due to the non-metallic section at the tip of a carbon nanotube, field penetration is expected to occur at the nanotube tip. Band bending due to field penetration in a high field, which can change the surface band structure, is thought to play an important role in field emission. The existing theory of band bending by field penetration in the surface spacecharge region of semiconductor was developed by Tsong [9] and Stratton [10]. The objectives of this study are to investigate the change in the electronic structure of carbon nanotubes due to field penetration into the nanotube tip by first-principle, quantum mechanical calculations. Nanotubes with different tip configurations and helical symmetries are studied.

\section{Theoretical approach and simulation models}

The calculations were performed using the CASTEP code [11,12], which is a plane-wave, pseudopotential program based on density functional theory (DFT). The generalized gradient approximation (GGA) [13] is used with the exchange-correlation potential of Wang and Perdew (PW91) [14]. The ion-electron interaction is modeled by the non-local real space [15], ultrasoft pseudopotential [16]. A plane-wave basis with a cut-off energy of $340 \mathrm{eV}$ and the $\Gamma$ point approximation are used for our calculations. In this study, we compare the electronic properties of six types of CNTs. They are the armchair $(5,5) /$ zigzag $(9,0)$ capped-nanotubes, the $(5,5) /(9,0)$ open-ended nanotubes and the $(5,5) /(9,0)$ closeended nanotubes with hydrogen termination. The armchair $(5,5)$ capped single-walled carbon nanotube is represented by an eight layer ( 80 atoms) stem with its mouth capped by a half of a $\mathrm{C}_{60}$ molecule. The dangling bonds at the other end are saturated by hydrogen atoms to avoid boundary effects, giving a final structure consisting of total 120 atoms. The zigzag $(9,0)$ capped-nanotube is constructed similarly with eight layers of carbon rings capped by a $\mathrm{C}_{30}$ molecule. These structures are then constructed within a tetragonal supercell with a lattice constant of $30 \AA$ along the $z$-axis to represent the vacuum slab and a separation of $15 \AA$ along the $x$ - and $y$-axes to avoid interaction between two adjacent nanotubes. These atomic coordinates are fully optimized at zero electric field until the force on each atom after relaxation is less than $0.005 \mathrm{eV} / \AA$. The optimized geometry of a capped $(5,5)$ nanotube shows that the atoms at the top pentagon have an average bond length of $1.44 \AA$ compared to $1.42 \AA$ at the side wall whereas the average bond length at the top of a capped $(9,0)$ tube is about $1.43 \AA$. The relaxed structure of the openended $(5,5)$ nanotube, as shown in Table 1 has a smaller diameter at its mouth with a shorter $\mathrm{C}-\mathrm{C}$ bond length of $1.23 \AA$ compared to $1.43 \AA$ before relaxation. The formation of triple bonds at the tube mouth is also seen in the morphology of multiwalled carbon nanotubes [17]. The relaxed structure for the openended $(9,0)$, on the other hand, does not show significant formation of triple bonds as seen in the $(5,5)$ open-ended nanotube with an average bond length of $1.39 \AA$, leaving dangling bonds at the tip. For the close-ended $(5,5)$ and $(9,0)$ nanotubes, the dangling bonds at the tip are saturated by hydrogen atoms and the relaxed structures show an average $\mathrm{C}-\mathrm{H}$ bond length of $1.08 \AA$.

To study field emission properties of the carbon nanotube, a uniform external electric field is applied along the tube axis by using a sawtooth-type potential, which is compatible with the periodic boundary condition. Structural changes are rather small even under an external electric field $\sim 0.75 \mathrm{~V} / \AA$. The local field 
Table 1

The effective workfunction and the amount of charge redistribution of CNTs with and without an external electric field

\begin{tabular}{llllll}
\hline & CAP- $(5,5)$ & Open- $(5,5)$
\end{tabular}

CAP- $(5,5)$ represents the capped CNT- $(5,5)$. Open- $(5,5)$ represents the open-ended CNT- $(5,5)$. Open + H- $(5,5)$ represents the closed-ended CNT- $(5,5)$. CAP- $(9,0)$ represents the capped CNT- $(9,0)$. Open- $(9,0)$ represents the open-ended CNT- $(9,0)$. Open $+\mathrm{H}-(9,0)$ represents the closed-ended CNT- $(9,0)$. The bond length of $\mathrm{C} \equiv \mathrm{C}$ at the mouth of the open-ended CNT- $(5,5)$ is $1.23 \AA$.

strength around the tip is higher than the external applied field due to the small dimension of the tube tip. This effect is more enhanced with the increasing aspect ratio of the carbon nanotube. We calculated the maximum field strength outside the capped-nanotube as a function of the external electric field strength. A near linear dependence can be found until the critical external applied field strength $\sim 0.75 \mathrm{~V} / \AA$ is applied. The maximum field strength outside the capped end of the carbon nanotube at this applied field is about $2.5 \mathrm{~V} / \AA$ due to the field enhancement at the tip. Beyond this critical field, electrons become unbound and the accumulation of electrons at the end of supercell was found. In this new regime, the present computational approach, which employs a bound basis state, is no longer suitable. In addition, the workfunction, which is an important parameter in describing field emission properties, has been investigated for various nanotubes. The workfunction $\Phi$ of a metal surface is usually defined as the energy needed to take an electron from the Fermi level $\mu$ to the vacuum level, by $\Phi=\varphi-\mu$, where $\varphi$ is the electrostatic potential caused by a surface dipole resulting from the spilling-out of electron density at the metal surface $[18,19]$. In contrast to solids, the surface of one CNT is modeled by its wall and tip due to its unique geometrical characteristics. Therefore, the difference in potential energy of one electron between the highest occupied molecular orbital (HOMO) and the vacuum level is defined as the corresponding "effective workfunction" which is different from the conventional definition of workfunction. The vacuum level in our calculations is defined as the average value of the potential energy distribution in the vacuum region over which the potential energy nearly comes to a constant value, as shown in Fig. 1. The potential energy curves along the tube axis under an external electric field are also shown in Fig. 1. The "effective workfunction" is then defined as the difference between the top of the potential energy curve and the HOMO state.

\section{Result and discussion}

The calculated workfunctions of various nanotubes with and without external electric fields are summarized in Table 1. Firstly, let us examine the electronic properties of different carbon nanotubes without an external electric field. The capped armchair $(5,5)$ nanotube is found to have a workfunction $4.78 \mathrm{eV}$ which shows a good agreement with the recent experimental measurements of single-walled carbon nanotube bundles by Suzuki et al. [20] using the ultraviolet photoemission spectroscopy, which gave a value of $4.8 \mathrm{eV}$. Although the $(5,5)$ armchair nanotube is metallic, the capped-nanotube is found to be semiconducting with an energy gap of $1.4 \mathrm{eV}$ between the HOMO and LUMO (lowest unoccupied molecular orbital). This is due to the capping by half of a $\mathrm{C}_{60}$ molecule. The effective workfunction of capped $(9,0)$ zigzag nanotube shows a lower value of $4.14 \mathrm{eV}$. For the open-ended $(5,5)$ nanotube, as mentioned above, the formation of triple bonds at the tip leads to the charge redistribution after structural relaxation, leading to a substantial decrease in the spilling-out of 


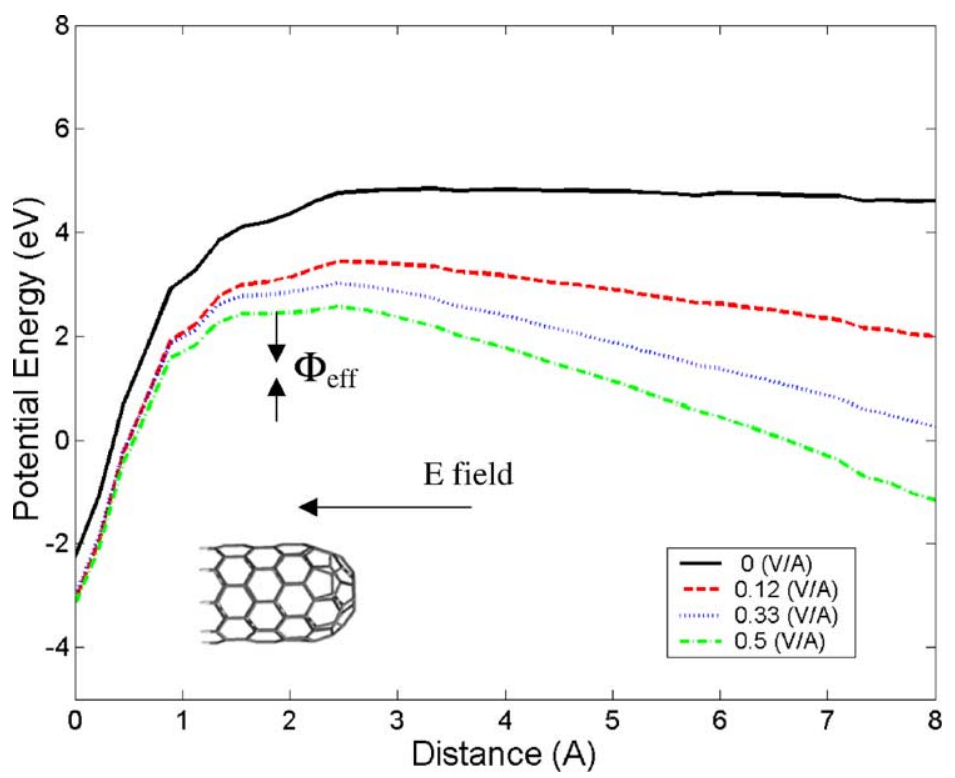

Fig. 1. Potential-energy curves for different external electric fields applied to a capped $(5,5)$ single-walled carbon nanotube tip. The Fermi energy is located at $0 \mathrm{eV}$. The effective workfunction is defined as the distance between the top of the potential profile and the Fermi energy.

electron density at the nanotube tip after structural relaxation, which reduces the surface dipole. This leads to a reduction of its workfunction with a lower value of $4.39 \mathrm{eV}$ compared to the $(5,5)$ cappednanotube of $4.78 \mathrm{eV}$. The open-ended $(9,0)$ nanotube, on the other hand, has unsaturated dangling bonds at its mouth, giving a substantial increase in the spillingout of electron density at the nanotube tip, which increases its surface dipole. It gives a workfunction of $5.1 \mathrm{eV}$. The close-ended $(5,5) \mathrm{CNT}$ exhibits a further reduction in its workfunction with a value of $3.75 \mathrm{eV}$ at zero electric field and the resulting lower workfunction can be attributed to the presence of a relatively small dipole on the $\mathrm{C}-\mathrm{H}$ bond, $\mathrm{C}^{\delta-}-\mathrm{H}^{\delta+}$, owing to the different electronegativity between $\mathrm{C}$ and $\mathrm{H}$. The potential difference across the dipole lowers the distance between the Fermi energy and the vacuum level, leading to a reduction of workfunction. The close-ended $(9,0)$ CNT also demonstrates a similar result with a lower workfunction value of $3.18 \mathrm{eV}$. Such enhanced field emission properties from CNTs by hydrogen termination have also been reported by the recent experiment carried by Zhi et al. [21] using hydrogen plasma treatment on CNTs. Similar phenomena are also seen in the $\mathrm{H}$-terminated diamond surface, which leads to its negative electron affinities [22].

$$
\begin{aligned}
& \Delta Q(\text { charge difference }) \\
& =Q(\text { with external field }) \\
& \quad-Q(\text { without external field })
\end{aligned}
$$

In the presence of an external electric field, the electrons along the tube axis are redistributed and this leads to the variation of the effective workfunction as well. Fig. 2 shows the charge redistribution along the capped $(5,5)$ nanotube axis under different applied electric fields. The magnitude of redistributed charge $\Delta Q$ is found to be nearly linear as a function of the applied external field strengths. It can be seen that the nanotube tube is now polarized, with an increased charge density at the tip end and a decreased charge density at the bottom end. The polarization can be mainly attributed to charge redistribution of $\pi$ electrons along the tube axis. The increase of charge density at the tip end leads to the occupations of the higher energy states, resulting in a change of the potential energy profile. The total potential energy variation in the system is mainly determined by two factors-the external potential and the electron-electron repulsion 

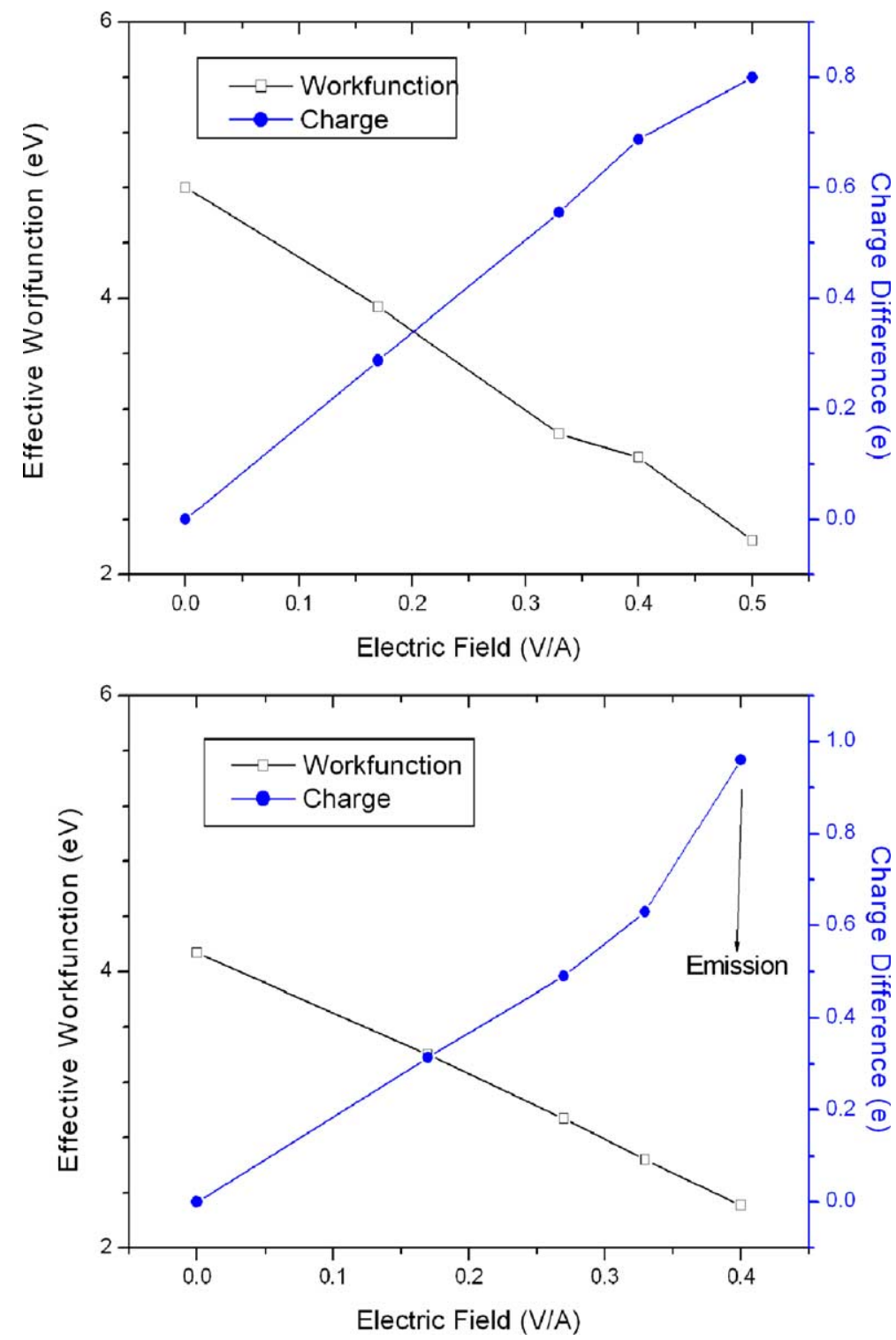

Fig. 2. Variations in the effective workfunction of capped $(5,5)$ and $(9,0)$ single-walled carbon nanotube tip and the corresponding charge redistribution as a function of applied electric field.

term induced by the accumulation of electrons at the tip. The degree of polarization along the tube axis is found to be strongly dependent on the applied field before the critical field is achieved. The effective workfunction defined as the difference between the Fermi level and the top of the potential profile, reduces with the applied field due to the accumulation of charge at the tube tip, which raises the Fermi level of the CNTs. The capped $(5,5)$ CNT has a higher workfunction value compared to the $(5,5)$ open-ended one under no applied field. With the external electric field, the capped $(5,5) \mathrm{CNT}$ now shows a lower value of workfunction than its $(5,5)$ open-ended counterpart due to a larger amount of charge redistribution to the 
capped end of CNTs. The atoms at the capped region are able to provide more states to accommodate the redistributed charge. The field penetration into the fullerene-like nanotube tip leads to a further reduction of the effective workfunction, which accounts for the ideal emission application of CNTs compared to that of the metallic tip regarded as a perfect conductor where no field penetration is observed. CNTs will therefore be excellent field emitter candidates with the combination of the metallic tube body and semiconducting tip end. The former being good for electron transport, the latter is good for electron emission due to the band bending occurring by field penetration. For the $(9,0)$ capped-nanotube, the charge redistribution

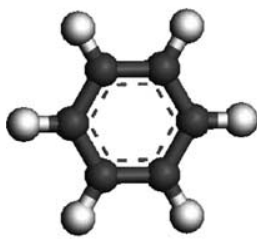

$\Delta \mathrm{Q}=0.202 \mathrm{e}$
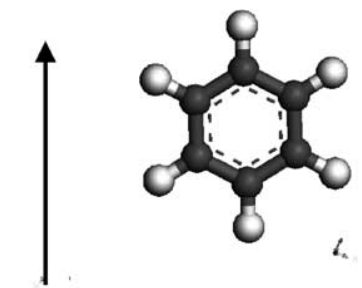

Field $=0.33 \mathrm{~V} / \AA$

$\Delta \mathrm{Q}=0.210 \mathrm{e}$

Fig. 3. Charge redistribution in two benzene rings with different orientations under an external electric field. $\Delta \rho=$ charge $(F=0.33 \mathrm{~V} / \AA)-$ charge $(F=0 \mathrm{~V} / \AA)$. The amount of charge redistribution for two benzene rings is $0.202 \mathrm{e}$ for armchair-like benzene and $0.210 \mathrm{e}$ for zigzag-like benzene, respectively.
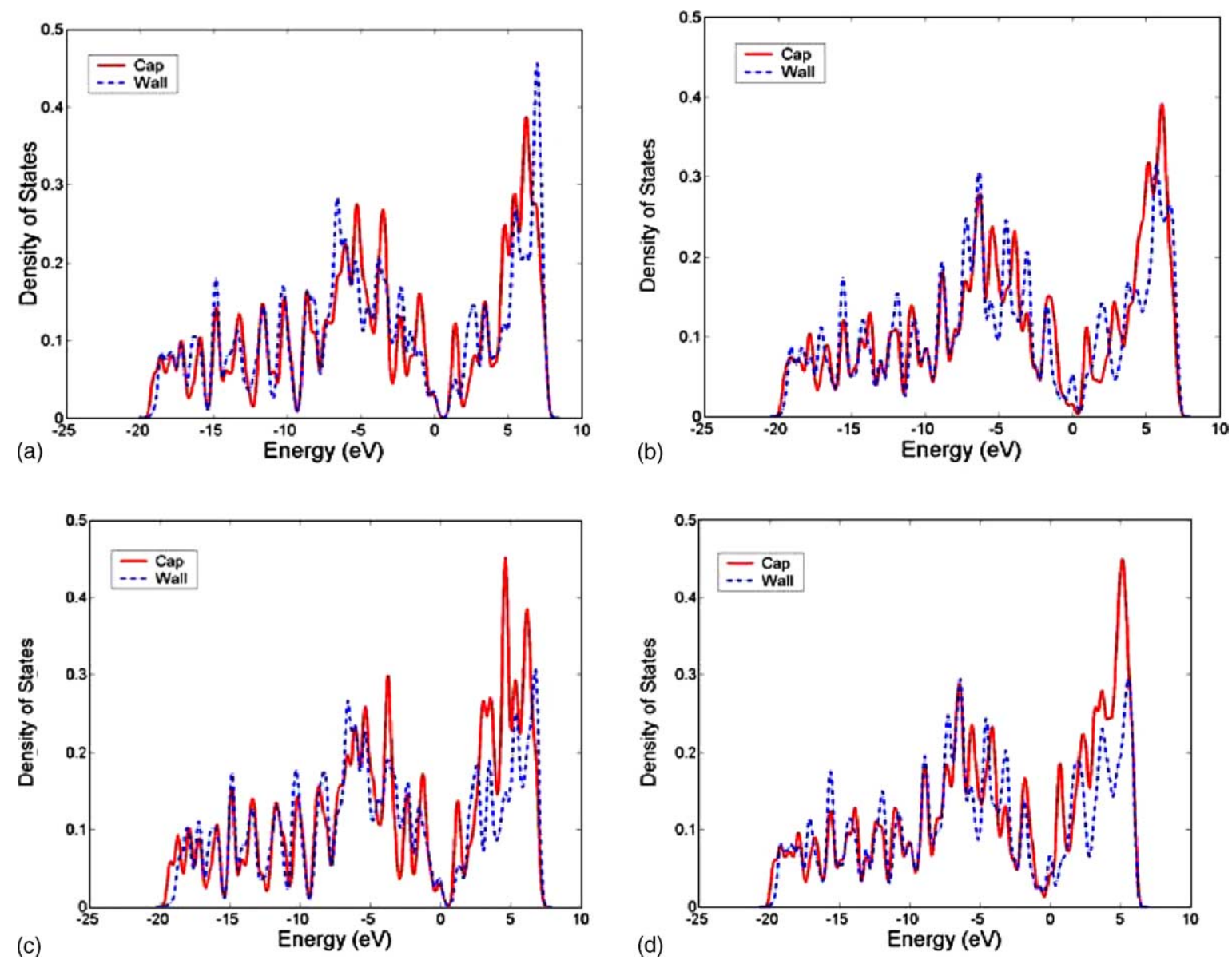

Fig. 4. The density of states (DOS) of the cap and sidewall parts of the capped armchair $(5,5)$ and zigzag $(9,0)$ carbon nanotubes with and without an external electric field, respectively. The ratios of DOS between that of the capped (a) and sidewall $(b)$ parts for $(5,5)$ and $(9,0)$ are 1.05:1 and 1.07:1, respectively, without external electric field. These values increase to 1.30:1 (c) and 1.41:1 (d) with an external electric field of $0.33 \mathrm{~V} / \AA$. 
and the degree of polarization are more pronounced, which leads to a lower effective workfunction than that of the $(5,5)$ capped-nanotube. This may be attributed to the different orientation of the carbon rings. We compare the charge redistribution of two different benzene rings under the same electric field as shown in Fig. 3. The armchair-like benzene ring shows a smaller degree of charge redistribution than the zigzag-like one. The charge redistribution of $\pi$ electrons along the zigzag-like tube axis is easier than in the armchair-like tube. Therefore, it is found that the capped-zigzag nanotubes show better field emission electronic properties than the capped $(5,5)$ counterpart. For the $(9,0)$ open-ended nanotube, although charge redistribution occurs under an external electric field, the high population of dangling bonds at the mouth causes strong electron-electron repulsion, which decreases the field-induced polarization. Fig. 4 shows the density of states (DOS) of capped armchair $(5,5)$ and zigzag $(9,0)$ nanotubes with and without external electric fields, respectively. For comparison, the intensities are then normalized by dividing the total number of atoms at the cap and sidewall parts, respectively. As the states near the Fermi level are believed to be responsible for field emission, we compare the DOS of cap and sidewall parts from $-5 \mathrm{eV}$ below $E_{\mathrm{f}}$ (Fermi level) to $5 \mathrm{eV}$ above $E_{\mathrm{f}}$. It is shown that under no external field, the capped region has a slightly higher DOS than the body region by about $5 \%$ for the $(5,5)$ armchair nanotube and $7 \%$ for the $(9,0)$ zigzag nanotube. Recent X-ray absorption spectroscopy experiments indicate that the DOS near $E_{\mathrm{f}}$ increases with the curvature of the CNT graphite sheet [23], not only from defect and dangling states. Under an external electric field of $0.33 \mathrm{~V} / \mathrm{A}$, the charge redistribution along the tube axis leads to significant variations of the DOS at the cap and sidewall parts. The DOS of the capped region for the $(5,5)$ nanotube is found to be about $30 \%$ higher than that of the sidewall part. The enhancement in the local density of states at the capped region is more pronounced for the $(9,0)$ nanotube where it is about $40 \%$ higher than that of the sidewall part. This result is consistent with the above finding that charge redistribution of $\pi$ electrons along the zigzag-like tube axis is easier than the armchair-like tube, leading to a higher DOS at the cap. Such enhancements of the DOS at the carbon nanotube tip show that electrons near the Fermi level will emit more easily due to the change of surface band structure resulting from the field penetration in a high field.

\section{Conclusion}

In summary, we have studied the field penetration induced charge redistribution effect on the field emission properties of carbon nanotubes by first-principle calculations. The effective workfunction is lowered under an external electric field due to charge redistribution at the surface. It is found that the carbon nanotube is polarized under the external electric field leading to charge redistribution. The band bending induced by field penetration into the nanotube tip surface can further reduce the effective workfunction due to the accumulation of charge at the tube tip, which raises the Fermi level of the CNTs. The magnitude of redistributed charge $\Delta Q$ is found to be nearly linear as a function of the applied external field strengths. In addition, we have found that the capped $(9,0)$ zigzag nanotube demonstrates better field emission properties than the capped $(5,5)$ nanotube because the charge redistribution of $\pi$ electrons along the zigzag-like tube axis is easier than in the armchairlike tube. The DOS of the capped region of the nanotube is found to be enhanced with a value of $30 \%$ higher than that of the sidewall part for the capped $(5,5)$ nanotube and $40 \%$ for the capped $(9$, $0)$ nanotube under the electric field of $0.33 \mathrm{~V} / \mathrm{A}$. Such enhancements of the DOS at the carbon nanotube tip show that electrons near the Fermi level will emit more easily due to the change of surface band structure resulting from the field penetration in a high field. CNTs will therefore be an excellent field emitter candidate with the combination of the metallic tube body and semiconducting tip end. The former being good for electron transport, the latter is good for electron emission due to the band bending occurring by field penetration.

\section{Acknowledgements}

This work is supported by National Science Council, Taiwan with the project number of NSC90-2112M155-002. 


\section{References}

[1] Q.H. Wang, A.A. Setlur, J.M. Lauerhaas, J.Y. Dai, E.W. Seelig, R.P.H. Chang, Appl. Phys. Lett. 72 (1998) 2912.

[2] C. Bower, W. Zhu, D. Shalom, D. Lopez, L.H. Chen, P.L. Gammel, S. Jin, Appl. Phys. Lett. 80 (2002) 3820.

[3] R.H. Folwer, L. Nordheim, Proc. R. Soc. London, Ser. A 119 (1928) 683.

[4] R. Saito, G. Dresselhaus, M.S. Dresselhaus, Physical Properties of Carbon Nanotubes, Imperial College Press, London, 1998.

[5] R. Tamura, M. Tsukada, Phys. Rev. B 52 (1995) 6015.

[6] P.G. Collins, A. Zettl, Phys. Rev. B 55 (1997) 9391.

[7] M.J. Frasen, Th.L. von Rooy, P. Kruit, Appl. Surf. Sci. 146 (1999) 312-327.

[8] S. Han, J. Ihm, Phys. Rev. B 66 (2002) 241402; C. Kim, B. Kim, S.M. Lee, C. Jo, Y.H. Lee, Phys. Rev. B 65 (2002) 165418.

[9] T.T. Tsong, Surf. Sci. 81 (1979) 28-42.

[10] R. Stratton, Phys. Rev. 135 (1964) A794.

[11] M.C. Pyane, M. Teter, D.C. Allan, J.D. Joannopoulos, Rev. Mod. Phys. 64 (1992) 1045.

[12] V. Mailman, B. Winkler, J.A. White, C.J. Pickard, M.C.
Payne, E.V. Akhmatskaya, R.H. Nobes, Int. J. Quantum Chem. 77 (2000) 895-910.

[13] J.P. Perdew, J.A. Chevary, S.H. Vosko, K.A. Jackson, M.R. Pederson, D.J. Singh, C. Fiolhais, Phys. Rev. B 46 (1992) 6671-6687.

[14] Y. Wang, J.P. Perdew, Phys. Rev. B 43 (1991) 8911;

Y. Wang, J.P. Perdew, Phys. Rev. B 44 (1991) 13298.

[15] R.D. King-Smith, M.C. Pyane, J.S. Lin, Phys. Rev. B 44 (1991) 13063.

[16] D. Vanderbilt, Phys. Rev. B 41 (1990) 7892-7895.

[17] Y.-K. Kwon, Y.H. Lee, S.-G. Kim, P. Jund, D. Tománek, R.E. Smalley, Phys. Rev. Lett. 79 (1997) 2065-2068.

[18] N.D. Lang, Phys. Rev. B 4 (1971) 4234.

[19] J. Robertson, Diamond Related Mater. 5 (1996) 791.

[20] S. Suzuki, C. Bower, Y. Watanabe, O. Zhou, Appl. Phys. Lett. 76 (2000) 4007.

[21] C.Y. Zhi, X.D. Bai, E.G. Wang, Appl. Phys. Lett. 81 (2002) 1690-1692.

[22] M.J. Rutter, J. Robertson, Phys. Rev. B 57 (1997) 9241.

[23] J.W. Chiou, C.Y. Yueh, J.C. Jan, H.M. Tasi, W.F. Pong, I.H. Hong, R. Klauser, M.H. Tsai, Y.K. Chang, Y.Y. Chen, C.T. Wu, K.H. Chen, S.L. Wei, C.Y. Wen, L.C. Chen, T.J. Chuang, Appl. Phys. Lett. 81 (2002) 4189. 